\title{
Paraganglioma gangliocítico como histología definitiva tras ampulectomía por sospecha de ampuloma
}

\section{Gangliocytic paraganglioma as definitive histology after ampullectomy due to suspected ampuloma}

\author{
Ana García-Vico ${ }^{1 *}$, Darío Martínez-Baena ${ }^{1}$, José M. Lorente-Herce ${ }^{1}$, Granada Jiménez-Riera ${ }^{1}$, \\ Jéssica Sánchez-Santos ${ }^{2}$ y Pablo Parra-Membrives ${ }^{1}$ \\ ${ }^{1}$ Servicio de Cirugía General y del Aparato Digestivo; ${ }^{2}$ Servicio de Anatomía patológica. Hospital Virgen de Valme, Sevilla, España
}

\begin{abstract}
Resumen
Antecedentes: El paraganglioma gangliocítico es un tumor raro que se localiza comúnmente en el duodeno. Caso clínico: Varón de 60 años con antecedentes de pancreatitis enólica crónica estudiado por síndrome constitucional. La tomografía computarizada evidenció una masa en la segunda rodilla duodenal, periampular. No pudo conseguirse el diagnóstico histológico al tratarse de una masa submucosa. Ante la sospecha de ampuloma se realizó una ampulectomía. Conclusión: El diagnóstico preoperatorio es difícil, al tratarse de un tumor de localización submucosa, lo que dificulta su estudio histológjco. Dada su baja probabilidad de metástasis y de recurrencia, se considera suficiente la escisión con márgenes libres.
\end{abstract}

Palabras clave: Paraganglioma gangliocítico. Ampuloma. Ampulectomía.

\begin{abstract}
Background: Gangliocytic paragangliomas are rare tumors most commonly located in the duodenum. Clinical case: $A$ 60-year-old male was studied for a constitutional syndrome. Given his history of chronic alcoholic pancreatitis, the main suspicion was a pancreatic carcinoma. Computed tomography scan showed a periampullary mass in the second portion of the duodenum. The pre-operative histological diagnosis couldn't be obtained due to the submucosal location of the mass. With the suspicion of an ampulloma, an ampullectomy was performed. Conclusion: Preoperative histological diagnosis is difficult since it is a submucosal mass. Due to the low probability of metastasis and recurrence, excision is the recommended procedure.
\end{abstract}

Key words: Gangliocytic paraganglioma. Ampulloma. Ampullectomy. DOI: 10.24875/RHJM.20000025
Disponible en internet: 13-11-2020 Rev Hosp Jua Mex. 2020;87(3):159-161

www.revistahospitaljuarez.com licencia CC BY-NC-ND (http://creativecommons.org/licenses/by-nc-nd/4.0/). 


\section{Antecedentes}

El paraganglioma gangliocítico es un tumor raro que se localiza en el duodeno en el $90 \%$ de los casos, con especial predilección por la ampolla de Vater ${ }^{1}$. Se han comunicado unos 200 casos desde la primera descripción de este tumor en 1957. La presentación clínica más común incluye melenas y dolor abdominal, pero lo más frecuente es que sea un hallazgo incidental en pruebas de imagen encontrándose el paciente asintomático².

Suelen tener un comportamiento benigno, con metástasis linfáticas regionales en el $5-7 \%$ de casos ${ }^{1}$. No se han reportado muertes por esta causa y la recurrencia tras resección es rara.

Es necesaria la confirmación histopatológica e inmunohistoquímica para su diagnóstico ${ }^{3}$. Su tratamiento es la escisión quirúrgica completa con márgenes libres para prevenir la recaída.

Presentamos un caso de paraganglioma gangliocítico a nivel de la ampolla de Vater en un paciente con antecedente de pancreatitis crónica y estudiado por síndrome constitucional.

\section{Caso clínico}

Varón de 60 años, fumador y exbebedor. Historia de pancreatitis crónica de origen alcohólico, con varios ingresos por reagudización y diabetes e insuficiencia exocrina secundaria. Colecistectomizado en 2014.

En seguimiento por Medicina Interna por síndrome constitucional y pérdida de peso de dos meses de evolución, sin otra sintomatología. Analíticamente sin hallazgos. Dados los antecedentes y la sospecha de posible neoplasia pancreática se practica tomografía computarizada (TC) de abdomen que describe una tumoración de $22 \mathrm{~mm}$ en segunda rodilla duodenal (Fig. 1), sospechosa de ampuloma. Para completar el estudio se realiza endoscopia digestiva alta en la que se observa masa en área papilar con mucosa suprayacente de características normales que se biopsia. La anatomía patológica muestra mucosa duodenal normal. Se realiza ecoendoscopia que muestra masa submucosa a nivel de papila de $14 \times 13 \mathrm{~mm}$ que infiltra hasta capa muscular propia tomando nuevamente biopsia a nivel de ampolla de Vater. Dichas biopsias informan de mucosa duodenal sin evidencia de malignidad.

Tras discutirse el caso en comité multidisciplinario y ante la alta sospecha de ampuloma, se decide intervención quirúrgica. Tras movilizarse el duodeno

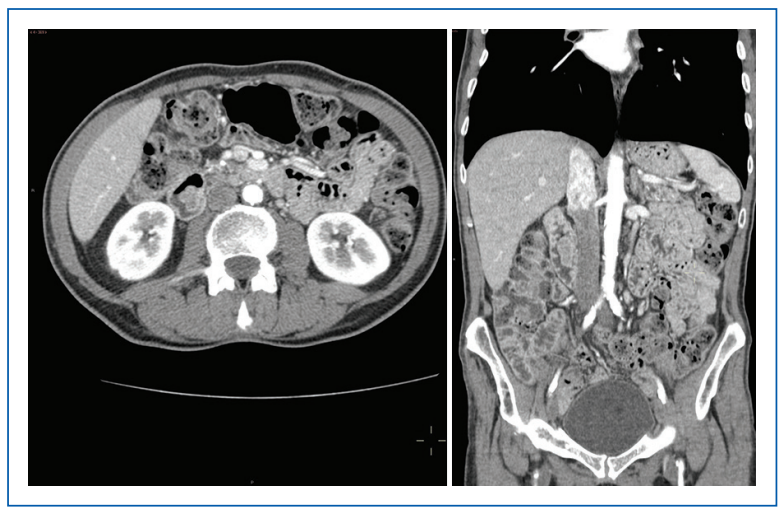

Figura 1. Flecha: masa en segunda porción duodenal. A: corte axial. B: corte coronal.

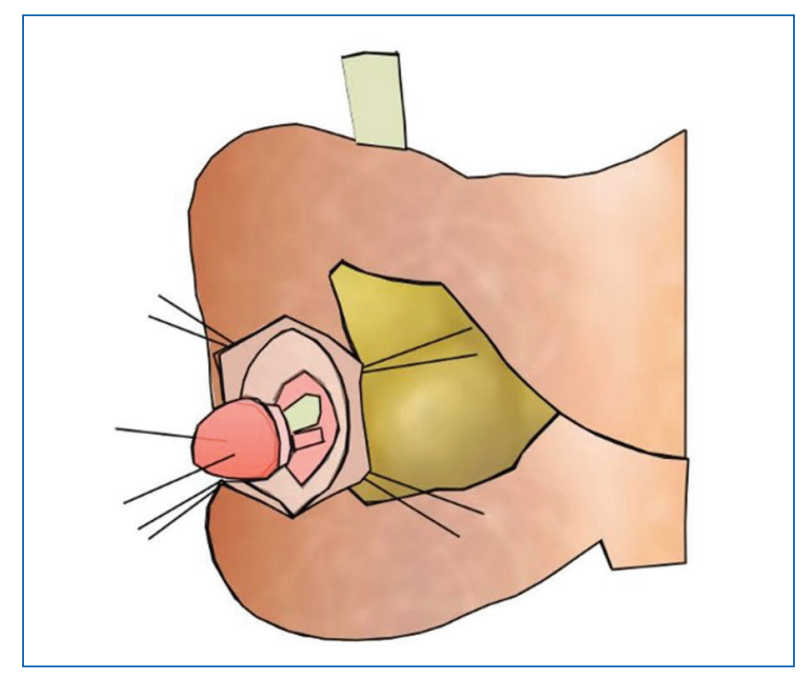

Figura 2. Esquema ampulectomía.

mediante maniobra de Kocher y apertura de este mediante duodenotomía longitudinal, se realiza escisión circular en bloque de ampolla de Vater incluyendo vía biliar y conducto pancreático distales. Se envía biopsia intraoperatoria informando de bordes libres de malignidad. Se realiza septoplastia uniendo vía biliar y Wirsung con monofilamento reabsorbible $5 / 0$ y posterior reimplantación en mucosa duodenal con puntos sueltos del mismo material (Fig. 2). Postoperatorio sin complicaciones, siendo dado de alta al séptimo día.

La anatomía patológica definitiva informa de paraganglioma gangliocítico que infiltra hasta capa muscular propia, sin evidencia de contacto de la lesión con los márgenes quirúrgicos (Fig. 3).

En control evolutivo posterior, TC sin evidencia de enfermedad y endoscopia con área cicatricial que se biopsia y muestra mucosa normal. 


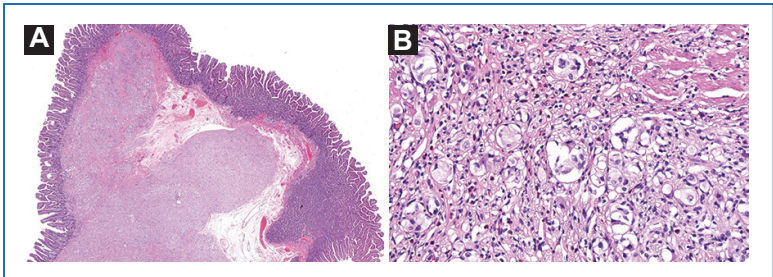

Figura 3. A: masa submucosa circunscrita con células fusiformes dispuestas en nidos y trabéculas, compatible con paraganglioma gangliocítico. B: imagen a mayor aumento en la que se identifican células ganglionares, sobre fondo de células fusiformes con aspecto neuroide.

\section{Discusión}

Los paragangliomas gangliocíticos son tumores raros, que Dahl, et al. describieron por primera vez en $1957^{4}$. Aunque se han descrito casos en otras localizaciones, el $90 \%$ asienta en la segunda porción del duodeno, con especial preferencia por la ampolla de Vater.

En la clasificación de tumores del aparato digestivo de la WHO (2000), los paragangliomas gangliocíticos se clasifican como tumores epiteliales ${ }^{2}$. La edad media de aparición es de 54 años (rango de edad de 17 a 83 años) ${ }^{4}$. La incidencia es levemente mayor en hombres.

Aunque suelen ser un hallazgo incidental en pacientes asintomáticos, cuando se reportan síntomas lo más frecuente es dolor abdominal y melenas. Según una revisión de 51 casos publicados en la literatura por Burke, et al., los síntomas reportados son dolor abdominal, sangrado gastrointestinal, melenas, anemia, obstrucción pilórica y biliar ${ }^{5}$.

Las características endoscópicas no difieren de otros tumores submucosos. Debido a esta localización, el diagnóstico histológico preoperatorio es difícil por biopsia endoscópica ${ }^{6}$.

El comportamiento de este tumor suele ser benigno, con un $5-7 \%$ de metástasis linfáticas regionales ${ }^{1}$. El tratamiento se basa en la escisión completa del tumor. Se ha descrito la vía endoscópica, pero normalmente se realiza por vía quirúrgica ${ }^{3}$. Por la baja probabilidad de metástasis y recurrencia se considera suficiente la escisión del tumor. En caso de afectación linfática regional está indicada la duodenopancreatectomía cefálica seguida de radioterapia ${ }^{4}$.

No se han descrito casos de recurrencia tras resección completa con bordes libres de la masa, por lo que no se indica adyuvancia en esos casos. No se ha descrito el empleo de quimioterapia para esta enfermedad ${ }^{1}$.

En este caso no se llegó al diagnóstico preoperatoriamente, las biopsias que se practicaron informaban de mucosa normal. Dada la sospecha de ampuloma se decidió practicar ampulectomía informando la anatomía patológica definitiva de paraganglioma gangliocítico sin afectación de bordes de resección.

\section{Financiamiento}

La presente investigación no ha recibido ayudas específicas provenientes de agencias del sector público, sector comercial o entidades sin ánimo de lucro.

\section{Conflicto de intereses}

Los autores declaran no tener conflicto de intereses alguno.

\section{Responsabilidades éticas}

Protección de personas y animales. Los autores declaran que para esta investigación no se han realizado experimentos en seres humanos ni en animales.

Confidencialidad de los datos. Los autores declaran que han seguido los protocolos de su centro de trabajo sobre la publicación de datos de pacientes.

Derecho a la privacidad y consentimiento informado. Los autores declaran que han obtenido el consentimiento informado de los pacientes y/o sujetos referidos en el artículo.

\section{Bibliografía}

1. Loftus TJ, Kresak JL, Gonzalo DH, Sarosi GA, Behrns KE. Duodenal gangliocytic paraganglioma: A case report and literature review. Int $J$ Surg Case Rep. 2015;8C:5-8.

2. Kwon J, Lee SE, Kang MJ, Jang J, Kim S. A case of gangliocytic paraganglioma in the ampulla of Vater. World J Surg Oncol. 2010;8:42.

3. Cruz-reyes JM, Pérez-corona T, Porras-escorcia O, Altamirano-arcos CA, Vargas-mejía JL, Godínez-franco LS. Paraganglioma gangliocítico del ámpula de Vater, resección endoscópica. Rev Hosp Jua Mex. 2015;82:173-6.

4. Sánchez-Pérez MA, Luque-de León E, Muñoz-Juárez M, Moreno-Paquentin E, Genovés-Gómez H, Torreblanca-Marín MA. FORMATION MÉDICALE CONTINUE Duodenal gangliocytic paraganglioma. Can J Surg. 2009;52(2):E27-8.

5. Burke AP, Helwig EB. Gangliocytic paraganglioma. Am J Clin Pathol. 1989;92(1):1-9.

6. Sánchez-Pobre P, Sáenz-López S, Rodríguez S, Sánchez F, Alemany I, López $\mathrm{G}$, et al. Safe endoscopic resection of gangliocytic paraganglioma of the major duodenal papilla. Rev Esp Enferm Dig. 2004;96(9):660-2; 663-4. 